T. Watanabe

Nagoya Math. J.

Vol. 37 (1970), 219-241

\title{
ON THE BOUNDARY GONDITION OF TRANSPORT SEMIGROUP
}

\section{TOITSU WATANABE}

\section{To Professor K. Ono on the occasion of his 60th birthday}

Let $S$ be the product space of finite interval $[-r, r]$ and two points $\Theta=\{\theta \pm 1\}$. We denote by $C(S)$ the Banach space of continuous functions on $S$ with norm $\|f\|=\max _{(x, \theta) \in S}|f(x, \theta)|$. Let $A$ be a differential operator of the first order defined on $S$ as the following:

$$
A u(x, \theta)=\theta a(x, \theta) \frac{d}{d x} u(x, \theta)-b(x, \theta) u(x, \theta)+c(x, \theta) u(x,-\theta) .{ }^{1)}
$$

A strongly continuous and positive contraction semigroup $\left\{T_{t}, t \geq 0\right\}$ on $C(S)$ is called transport semigroup if its infinitesimal generator is a suitable restriction of $A$.

The purpose of this paper is to study the question of the most general supplementary condition which restricts the given operator $A$ to a infinitesimal genereator of a transport semigroup. We first try to find the lateral (boundary) condition which is satisfied by all smooth functions in the domain of the infinitesimal generator of the given transport semigroup, following the method in A.D. Wentzell [14] (Theorem 1.1). We next show that such a condition is sufficient to determine the semigroup whose infinitesimal generator is a restriction of $A$ (Theorem 5.2).

Similar problems have been studied by W. Feller [3]-[6] in case of onedimensional diffusion (see also K. Ito, H.P. Mckean. Jr [10] and A.D. Wentzell [16]), and by P. Courrege [1], N. Ikeda [8], K. Sato, T. Ueno [11] and A.D. Wentzell [14]-[15] in case of multi-dimensional diffusion.

In $\$ 1$ of this paper, we show that any continuously differentiable function belonging to the domain of the infinitesimal generator satisfies the following boundary condition:

Received February 10, 1969

1) Precise definition is given in $\$ 1$. 
$L u(r \theta, \theta)=0, \quad(r \theta, \theta)$ is a boundary point of $S:$

$$
\begin{aligned}
L u(r \theta, \theta) & =r(r \theta, \theta) u(r \theta, \theta)+\delta(r \theta, \theta) A u(r \theta, \theta) \\
& +\mu(r \theta, \theta) \frac{\partial}{\partial n} u(r \theta, \theta) \\
& +\int_{S}[u(y, \vartheta)-u(r \theta, \theta)] \nu_{(r \theta, \theta)}(d(y, \vartheta)) .{ }^{1)}
\end{aligned}
$$

In section 2, we define the Green operator $G_{\alpha}^{\mathrm{min}}$ of the transport process with absorbing barriers and the harmonic operator $H_{\alpha}$ of $A$. We, in section 3 , give the semigroup $S_{t}^{\alpha}$ on Banach space $C(\partial S)$, consisting of all of functions on the boundary $\partial S=\{(r, 1)\} \cup\{(-r,-1)\}$, whose infinitesimal generator is $L H_{\alpha}$. In section 4 , we construct analytically the transport process with regular boundary (see Section 2) which is determined by $A$ and $L$, combining the Green operator $G_{\alpha}^{\text {min }}$ and the semigroup $S_{t}^{\alpha}$ on $C(\partial S)$, similarly to K. Sato and T. Ueno [11]. In section 5, we investigate the transport process with non-regular boundary.

Appendix is devoted to the investigation of the convergence of transport process to Brownian motion under some additional assumptions.

The author wishes to express his hearty thanks to Professor H. Kunita who gave him various advice and constant encouragement.

§1. Wentzell's boundary condition. Let $S$ be the product space of finite interval $[-r, r]$ and the two points set $\theta=\{\theta= \pm 1\}, \partial S$ be the boundary of $S$ which consists of two points $\{(r \theta, \theta): \theta= \pm 1\}$. Put $S^{0}=S-\partial S$, $=[-r, r) \times\{1\} \cup(-r, r] \times\{-1\}$. Let $C(S)$ be the space of all real valued continuous functions defined on $S$ with morm $\|f\|=\max _{(x, \theta) \in S}|f(x, \theta)|$. We call a system of linear operators $\left\{T_{t}, t \geq 0\right\}$ acting on $C(S)$ a strongly continuous and positive contraction semigroup, or simply a semigroup, on $C(S)$ if it satisfies $T_{t} T_{s}=T_{t+s}, T_{0}=$ the identity operator, $\left\|T_{t}\right\| \leq 1, \lim _{t \rightarrow 0}\left\|T_{t} f-f\right\|=0$ for any $f \in C(S)$, and $T_{t} f \geq 0$ for any $f \geq 0$. The infinitesimal generator (s) of a semigroup $\left\{T_{t}, t \geq 0\right\}$ is defined for such $f$ that the right hand side of

$$
\text { (3) } \left.f=\lim _{t \rightarrow 0} \frac{1}{t}\left(T_{t} f-f\right)^{2}\right)
$$

2) By $\lim _{n \rightarrow \infty} u_{n}=u$ or $u_{n} \rightarrow u$, we mean $\lim _{n \rightarrow \infty}\left\|u_{n}-u\right\|=0$. 
exists. The domain of $\mathbb{B S}$ is denoted by $\mathfrak{D}(\mathscr{S})$.

Let a in $C(S)$ be a function, non-negative on $S$ and positive in $S^{0}$. Define the monotone function $m(x, \theta)$ by:

$$
m(x, \theta)=\int_{0}^{x} \frac{d \xi}{\theta a(\xi, \theta)}, \quad-r \leq x \leq r .
$$

Let $C_{1(m)}(S)$ be the subspace of $C(S)$ consisting of function whose derivative with respect to $m$ also belongs to $C(S)$, i.e.,

$$
C_{1(m)}(S)=\left\{f \in C(S): \frac{d}{d m} f \in C(S)\right\}^{3)}
$$

REMARK. If $a \neq 0$ at $(x, \theta) \in S$, then $\frac{d}{d m} f(x, \theta)=\theta a(x, \theta) \frac{d}{d x} f(x, \theta)$.

We now define the differential operator $A$ on $C_{1(m)}(S)$ as follows:

$$
A u(x, \theta)=\frac{d}{d m} u(x, \theta)-b(x, \theta) u(x, \theta)+c(x, \theta) u(x,-\theta), \quad(x, \theta) \in S,
$$

where $b$ and $c$ are non-negative functions in $C(S)$ such that $b \geq c$.

We introduce the boundary condition as the following:

$$
\begin{aligned}
L u(r \theta, \theta) & =r(r \theta, \theta) u(r \theta, \theta)+\delta(r \theta, \theta) A u(r \theta, \theta)+\mu(r \theta, \theta) \frac{\partial}{\partial n} u(r \theta, \theta) \\
+ & \left.\int_{S}[u(y, \vartheta))-u(r \theta, \theta)\right]_{(r \theta, \theta)}(d(y, \vartheta))=0, \quad(r \theta, \theta) \in \partial S .
\end{aligned}
$$

Here $\gamma(r \theta, \theta) \leq 0, \delta(r \theta, \theta) \leq 0, \mu(r \theta, \theta) \geq 0, \frac{\partial}{\partial n} u(r \theta, \theta)=-\frac{d}{d m} u(r \theta, \theta), \nu_{(r \theta, \theta)}(E)$ is a $\sigma$-finite measure on $S$ such that

$$
\begin{aligned}
& \left.\nu_{(r \theta, \theta)}(\{r \theta, \theta)\}\right)=0, \\
& \nu_{(r \theta, \theta)}\left(S-U_{(r \theta, \theta)}\right)<\infty, \\
& \int_{U_{(r \theta, \theta)}} \xi_{(r \theta, \theta)}(y, \vartheta) \nu_{(r \theta, \theta)}(d(y, \vartheta))<\infty,
\end{aligned}
$$

where $U_{(r \theta, \theta)}$ is a neiborhood of the point $(r \theta, \theta)$ and

$$
\xi_{(r \theta, \theta)}(y, \vartheta)=\left\{\begin{array}{lll}
m(r \theta, \theta)-m(y, \theta) & \text { if } & \vartheta=\theta \\
1 & \text { if } \vartheta \neq \theta
\end{array}\right.
$$

3) $\frac{d}{d m} f(r \theta, \theta)=\lim _{(x, \theta) \rightarrow(r \theta, \theta)} \frac{d}{d m} f(x, \theta)$. 
Further if $r(r \theta, \theta)=\delta(r \theta, \theta)=\mu(r \theta, \theta)=0$, then $\nu_{(r \theta, \theta)}(S-\{(r \theta, \theta)\})=\infty$.

Then we have the following

Theorem 1. 1. Suppose that $|m(r \theta, \theta)|<\infty$ and $T_{t}$ is a (transport) semigroup with an infinitesimal generator is which is a restriction of $A$. Then there is a condition of type (1.2) which is satisfied at $(r \theta, \theta)$ by all functions $u \in \mathfrak{D}(\mathbb{S})$.

Proof. For $u \in \mathscr{D}(\mathscr{S})$, we have, at $(r \theta, \theta) \in \partial S$ such that $|m(r \theta, \theta)|<\infty$, (1. 4)

$$
\begin{aligned}
A u(r \theta, \theta) & =\operatorname{ds} u(r \theta, \theta) \\
& =\lim _{t \rightarrow 0} \frac{1}{t}\left(T_{t} u(r \theta, \theta)-u(r \theta, \theta)\right) \\
& =\lim _{t \rightarrow 0} \frac{1}{t}\left(\int_{S} u(y, \vartheta) P(t,(r \theta, \theta), d(y, \vartheta)-u(r \theta, \theta))\right. \\
& =\lim _{t \rightarrow 0}\left[r(t) u(r \theta, \theta)+\int_{S} \frac{u(y, \vartheta)-u(r \theta, \theta)}{\xi_{(r \theta, \theta)}(y, \vartheta)} \beta(t) N(t, d(y, \vartheta))\right],
\end{aligned}
$$

where $\gamma(t)=\frac{1}{t}(P(t,(r \theta, \theta), S)-1) \leq 0$,

$$
\begin{aligned}
\beta(t) & =\frac{1}{t} \int_{S} \xi_{(r \theta, \theta)}(y, \vartheta) P(t,(r \theta, \theta), d(y, \vartheta)), \\
N(t, E) & =\frac{1}{t \beta(t)} \int_{E} \xi_{(r \theta, \theta)}(y, \vartheta) P(t,(r \theta, \theta), d(y, \vartheta)), \quad E \in B(S)^{4)} .
\end{aligned}
$$

Putting

$$
v(y, \vartheta)=\frac{u(y, \vartheta)-u(r \theta, \theta)}{\xi_{(r \theta, \theta)}(y, \vartheta)},
$$

we have

$$
v(y, \vartheta)=\frac{\partial}{\partial n} u(r \theta, \theta)+\frac{R(u,(y, \vartheta))}{\xi_{(r \theta, \theta)}(y, \vartheta)}
$$

$R(u,(y, \vartheta))$ being continuous on $S-\{(r \theta, \theta)\}$ and $o\left(\xi_{(r \theta, \theta)}(y, \vartheta)\right)$ around $(r \theta, \theta)$, the second summand of $v(y, \vartheta)$ can be continuouly extended on $S$.

Formula (1.4) may now be rewritten in the form

$$
A u(r \theta, \theta)=\lim _{t \rightarrow 0}\left[\gamma(t) u(r \theta, \theta)+\beta(t) \int_{S} v(y, \vartheta) N(t, d(y, \vartheta))\right] .
$$

4) $B(S)$ is the topological Borel field of $S$. 
Let us select a sequence $\left\{t^{(k)}\right\}$ converging to zero so that we have the following: a) the sequences $\left\{\gamma\left(t^{(k)}\right)\right\},\left\{\beta\left(t^{(k)}\right)\right\}$ converge and $\left.b\right)$ the sequences of measures of $N\left(t^{(k)}\right)$ weakly converges to a measure $N$; i.e.,

$$
\int_{S} f(y, \vartheta) N\left(t^{(k)}, d(y, \vartheta)\right) \rightarrow \int_{S} f(y, \vartheta) N(d(y, \vartheta))
$$

for any function $f \in C(S)$. If both of the limits of $\left\{r\left(t^{(k)}\right)\right\},\left\{\beta\left(t^{(k)}\right)\right\}$ are finite, then we obtain a boundary condition of the form:

$$
A u(r \theta, \theta)=\gamma u(r \theta, \theta)+\beta \int_{S} v(y, \vartheta) N(d(y, \vartheta)) .
$$

But if not both of these sequences have not finite limits, then one of them tends to infinity faster than the other. If we divide the expression after the "lim" sign in (1.5) by its absolute value, then we obtain a boundary condition of the form:

$$
0=\gamma u(r \theta, \theta)+\beta \int_{S} v(y, \vartheta) N(d(y, \vartheta)) .
$$

We must yet rewrite these conditions so that they will be of the form (1. 2). To do this it is sufficient to put $r(r \theta, \theta)=r \leq 0, \mu(r \theta, \theta)=\beta N(\{(r \theta, \theta)\}) \geq 0$, and $\delta(r \theta, \theta)=1$ or 0 , depending on whether formula (1.6) or (1.7) holds:

$$
\left.\nu_{(r \theta, \theta)}(E)=\beta \int_{E-\{(r \theta, \theta)\}} \frac{1}{\xi_{(r \theta, \theta)}(y, \vartheta)} N(d y, \vartheta)\right) .
$$

The fact that the measure $\beta \cdot N$ is non-negative and finite guarantees that the measure $\nu_{(r \theta, \theta)}$ is non-negative and that condition (1.3) is fulfilled. We prove that the condition obtained does not take the form $0=0$. In fact, if

$$
r(r \theta, \theta)=\delta(r \theta, \theta)=\nu_{(r \theta, \theta)}(S-\{(r \theta, \theta)\})=0,
$$

then $\beta>0$ and condition (1.7) has the form:

$$
\int_{\{(r \theta, \theta)\}} v(y, \vartheta) N(d(y, \vartheta))=0 .
$$

Since

$$
N(S)=\int_{S} 1 \cdot N(d(y, \vartheta))=\lim _{k \rightarrow \infty} \int_{S} 1 \cdot N\left(t^{(k)}, d(y, \vartheta)\right)=1,
$$

we have

$$
\mu(r \theta, \theta)=\beta \int_{\{(r \theta, \theta)\}} N(d(y, \vartheta))=\beta>0 .
$$


Thus the form $0=0$ does not take place.

We finally prove that, if $\gamma(r \theta, \theta)=\delta(r \theta, \theta)=\mu(r \theta, \theta)=0$, then $\nu_{(r \theta, \theta)}(S-\{(r \theta, \theta)\})=\infty$. If it is not true, then we have

$$
u(r \theta, \theta)=\frac{1}{\nu_{(r \theta, \theta)}(S)} \int_{S} u(y, \vartheta) \nu_{(r \theta, \theta)}(d(y, \vartheta)) .
$$

Taking $u=\alpha G_{\alpha} f(f \in C(S))$, where $G_{\alpha}$ is the Green operator of $T_{t}$, and so letting $\alpha \rightarrow \infty$, we have

$$
f(r \theta, \theta)=\frac{1}{\nu_{(r \theta, \theta)}(S)} \int_{S} f(y, \vartheta) \nu_{(r \theta, \theta)}(d(y, \vartheta)),
$$

since $\alpha G_{\alpha} f \rightarrow f$. Hence, $\left(\nu_{(r \theta, \theta)}(S)\right)^{-1} \nu_{(r \theta, \theta)}$ is the delta measure with mass 1 only at $(r \theta, \theta)$, which contradicts the definition of $\nu_{(r \theta, \theta)}$. Therefore $\nu_{(r \theta, \theta)}(S)=\infty$. Thus it has been proved.

§2. Minimal Green operator and harmonic operator. We first classify the boundary point $(r \theta, \theta)$ as follows:

$(r \theta, \theta)$ is regular, if $|m(r \theta, \theta)|<\infty$ and $|m(r \theta,-\theta)|<\infty$ :

$(r \theta, \theta)$ is exit, $\quad$ if $|m(r \theta, \theta)|<\infty$ and $|m(r \theta,-\theta)|=\infty$ :

$(r \theta, \theta)$ is entrance, if $|m(r \theta, \theta)|=\infty$ and $|m(r \theta,-\theta)|<\infty$ :

$(r \theta, \theta)$ is natural, if $|m(r \theta, \theta)|=\infty$ and $|m(r \theta, \theta)|=\infty$.

Let us define the differential operator on $S$ by

$$
A \cdot u(x \theta)=\frac{d}{d m} u(x, \theta)-b(x, \theta) b(x, \theta),
$$

where $m$ and $b$ are in (1. 1).

Then we have

Lemma 2. 1. i) For any constant $\alpha \geq 0$ and $f \in C(S)$, the solution $u$ in $C_{1(m)}(S)$ of

$$
\begin{aligned}
\left(\alpha-A^{*}\right) u(x, \theta)=f(x, \theta), & (x, \theta) & \in S^{0} \\
u(r \theta, \theta)=0, & (r \theta, \theta) & \in \partial S .
\end{aligned}
$$

exists. Such $u$ is unique and we denote it by $G_{a}^{*} f$.

ii) $G_{\alpha}^{\cdot}$ is linear, non-negative and bounded; $\left\|G_{\alpha}^{*}\right\| \leq 1 / \alpha \quad(\alpha>0)$.

iii) For any $f \in C(S)$ and $(x, \theta) \in S^{0}$, 


$$
\lim _{\alpha \rightarrow \infty} \alpha G_{\alpha} f(x, \theta)=f(x, \theta) .
$$

Moreover, if $f$ vanishes on $\partial S$,

$$
\lim _{\alpha \rightarrow \infty} \alpha G_{\alpha} f=f \text {. }
$$

iv) For $\alpha, \beta \geq 0$,

$$
G_{\dot{\alpha}}-G_{\dot{\beta}}+(\alpha-\beta) G_{\dot{\alpha}} G_{\dot{\beta}}=0 .
$$

v) If a boundary point $(r \theta, \theta)$ is exit or natural,

$$
G_{\alpha} f(r \theta,-\theta)=0 .
$$

If $(r \theta, \theta)$ is entrance or natural,

$$
G_{\alpha} f(r \theta, \theta)=0 .
$$

Proof. It is known (e.g. [7]) that we have

$$
G_{\alpha} f(x, \theta)=\int_{x}^{r \theta} \exp \left\{-\int_{x}^{y} \frac{\alpha+b(\xi, \theta)}{\theta a(\xi, \theta)} d \xi\right\} f(y, \theta) \frac{d y}{\theta a(y, \theta)}
$$

and the Green operator $G_{\alpha}$ have the properties i) - v).

Lemma 2. 2. The operator $A$ with domain $C_{1(m)}(S)$ is closed in $C(S)$.

Proof is easily obtained by noting that $A u(x, \theta)=f(x, \theta)$ is equivalent to

$$
\begin{gathered}
u(x, \theta)=u(0, \theta)+\int_{0}^{x} \frac{1}{\theta a(y, \theta)}[b(y, \theta) u(y, \theta)-c(y, \theta) u(y,-\theta) \\
+f(y, \theta)] d y .
\end{gathered}
$$

Theorem 2. 1. i) For any constant $\alpha>0$ and $f \in C(S)$, the solution in $C_{1(m)}(S)$ of

$$
\begin{array}{ll}
(\alpha-A) u(x, \theta)=f(x, \theta), & (x, \theta) \in S^{0} \\
u(r \theta, \theta)=0, & (r \theta, \theta) \in \partial S
\end{array}
$$

exists and such $u$ is unique. We denote it by $G_{\alpha}^{\min } f$.

ii) $G_{\alpha}^{\mathrm{min}}$, considered as an operator in $C(S)$, is linear, nonnegative and bounded; $\left\|G_{\alpha}^{\min }\right\| \leq 1 / \alpha(\alpha>0)$.

iii) For any $f \in C(S)$ and $(x, \theta) \in S^{0}$,

$$
\lim _{\alpha \rightarrow \infty} \alpha G_{\alpha}^{\min } f(x, \theta)=f(x, \theta) .
$$


Further, the convergence is uniform in $(x, \theta) \in S$, if $f$ vanishes on $\partial S$.

iv) For $\alpha, \beta>0$,

$$
G_{\alpha}^{\min }-G_{\beta}^{\min }+(\alpha-\beta) G_{\alpha}^{\min } G_{\beta}^{\min }=0 .
$$

v) If a boundary $(r \theta, \theta)$ point is exit or natural, then

$$
G_{\alpha}^{\min } f(r \theta,-\theta)=0 .
$$

If $(r \theta, \theta)$ is entrance or natural, then

$$
G_{\alpha}^{\min } f(r \theta, \theta)=0 \text {. }
$$

Proof. Putting $U f(x, \theta)=c(x, \theta) f(r,-\theta)$, we define $G_{\alpha}^{\min }$ by

$$
\begin{aligned}
G_{\alpha}^{\min } f & =G_{\dot{\alpha}} f+G_{\dot{\alpha}} U G_{\dot{\alpha}} f+G_{\alpha}^{\cdot} U G_{\alpha}^{\cdot} U G_{\dot{\alpha}} f+\cdots \\
& =\sum_{n=0}^{\infty} G_{\alpha}\left(U G_{\dot{\alpha}}\right)^{(n)} f^{5)},
\end{aligned}
$$

Then $G_{\alpha}^{\mathrm{min}}$ is well-defined for $\alpha>\|C\|$ and has properties ii), iii) and v), except that $\left\|G_{\alpha}^{\min }\right\| \leq 1 / \alpha$. Because of the closedness of $A \wedge \sim_{\min } \mathbf{A}_{\ldots} \ldots 11$ defined and

$$
\begin{aligned}
& (\alpha-A) G_{\alpha}^{\min } f \\
= & \lim _{N \rightarrow \infty}(\alpha-A)\left[\sum_{n=0}^{N} G_{\alpha}^{\cdot}\left(U G_{\dot{\alpha}}^{\cdot}\right)^{(n)}\right] f \\
= & \lim _{N}\left[\sum_{n=0}^{N}\left(U G_{\alpha}^{\cdot}\right)^{(n)} f-U G_{\alpha} \cdot\left(\sum_{n=0}^{N}\left(U G_{\alpha}^{\cdot}\right)^{(n)} f\right)\right] \\
= & \lim _{N}\left[f-\left(U G_{\alpha}^{\cdot}\right)^{(n+1)} f\right]=\dot{f} .
\end{aligned}
$$

Hence $G_{\alpha}^{\min } f$ is a solution of (2. 4).

To prove the uniqueness of the solution, we have only to show that $w=v-G_{\alpha}^{\min } f$ becomes identically zero, if $v$ is an another solution. Since $w$ satisfies the following:

$$
\begin{array}{ll}
(\alpha-A) w(x, \theta)=0, & (x, \theta) \in S^{0} \\
w(r \theta, \theta)=0, & (r \theta, \theta) \in \partial S,
\end{array}
$$

and $\alpha-A=\alpha-A^{\cdot}-U$, we have $w=G_{\alpha}^{\cdot} U w$. This leads us $w=\left(G_{\alpha}^{*} U\right)^{(n)} w$. Therefore $\|w\| \leq\left\|\left(G_{\alpha}^{\cdot} U\right)^{(n)} w\right\| \leq(\|c\| / \alpha)^{n}\|w\| \rightarrow 0$, and so $w=0$.

5) $\left(U G_{\dot{\alpha}}\right)^{(n)}$ means the $n$-fold operation of $\left(U G_{\dot{\alpha}}\right)$. 
It follows from the positive maximum principle of $A$ that

$$
\begin{aligned}
& \max _{(x, \theta) \in S} \alpha G_{\alpha}^{\min } 1(x, \theta)=\alpha G_{\alpha}^{\min } 1\left(x_{0}, \theta_{0}\right) \\
& =1+A G_{\alpha}^{\min 1} 1\left(x_{0}, \theta_{0}\right) \\
& \leq 1 \text {, }
\end{aligned}
$$

i.e., $\left\|G_{\alpha}^{\min }\right\| \leq 1 / \alpha(\alpha>\|c\|)$. By Hille-Yosida's Theorem, $G_{\alpha}^{\min }$ can be extended for all $\alpha>0$ and so has properties ii) $-v$ ). Thus we complete the proof.

To construct the harmonic function of $(\alpha-A)$, we prepare a following lemma.

Lemma 2. 3. i) For $\alpha>0$ and $\varphi \in C(\partial S)^{6)}$ such that $\varphi(r \theta, \theta)=0$ if $(r \theta, \theta)$ is an exit or natural boundary, the solution $u$ in $C_{1(m)}(S)$ of

$$
\begin{aligned}
\left(\alpha-A^{\cdot}\right) u(x, \theta)=0, & (x, \theta) \in S^{0}, \\
u(r \theta, \theta)=\varphi(r \theta, \theta), & (r \theta, \theta) \in \partial S
\end{aligned}
$$

uniquely exists. We denote it by $H_{\alpha}^{\cdot} \varphi$.

ii) $H_{\alpha}$ is linear, non-negative and bounded; $\left\|H_{\alpha}^{\cdot}\right\| \leq 1$.

iii) If $(r \theta, \theta)$ is an exit or natural boundary, then

$$
H_{\alpha}^{\cdot} \varphi(r \theta,-\theta)=0 .
$$

If $(r \theta, \theta)$ is entrance or natural, then.

$$
H_{\dot{\alpha}} \varphi(r \theta, \theta)=0 .
$$

Proof. As is well-known, $H_{\alpha}^{\cdot} \varphi(x, \theta)$ is given by

$$
\varphi(r \theta, \theta) \exp \left[-\int_{x}^{r \theta} \frac{\alpha+b(y, \theta)}{\theta a(y, \theta)} d y\right]
$$

which has the properties i) - iii).

Theorem 2.2. i) For any $\alpha>0$ and $\varphi \in C(\partial S)$ such that $\varphi(r \theta, \theta)=0$ if $(r \theta, \theta)$ is an entrance or natural boundary, the solution $u$ in $C_{1(m)}(S)$ of

$$
\begin{array}{ll}
(\alpha-A) u(x, \theta)=0, & (x, \theta) \in S^{0} \\
u(r \theta, \theta)=\varphi(r \theta, \theta), & (r \theta, \theta) \in \partial S
\end{array}
$$

6) $C(\partial S)$ is the space of bounded functions on $\partial S$ with sup-norm. 
exists. Such $u$ is unique and we denote it by $H_{\alpha}$.

ii) $H_{\alpha}$, considered as a mapping from $C(\partial S)$ into $C(S)$, is linear, non-negative and bounded; $\left\|H_{\alpha}\right\| \leq 1$.

iii) $H_{\alpha} \varphi$ does not take a positive maximum in $S^{0}$.

iv) If $(r \theta, \theta)$ is exit or natural, then $H_{\alpha} \varphi(r \theta,-\theta)=0$.

If $(r \theta, \theta)$ is entrance or natural, then $H_{\alpha} \varphi(r \theta, \theta)=0$.

Proof. Assigning

$$
\begin{aligned}
& H_{\alpha} \varphi=H_{\alpha} \varphi+G_{\alpha}^{\min } U H_{\alpha} \varphi \text { for } \varphi \in C(\partial S) \text {, we have } \\
& (\alpha-A) H_{\alpha} \varphi=\left(\alpha-A^{\cdot}-U\right) H_{\alpha}^{\cdot} \varphi+(\alpha-A) G_{\alpha}^{\min U} H_{\alpha}^{\cdot} \varphi \\
& =-U H_{\alpha}^{\dot{\alpha}} \varphi+U H_{\dot{\alpha}} \varphi=0
\end{aligned}
$$

and

$$
H_{\alpha} \varphi(r \theta, \theta)=H_{\dot{\alpha}} \varphi(r \theta, \theta)=\varphi(r \theta, \theta) .
$$

Hence, $H_{\alpha} \varphi$ is a solution of (2.8). Let $v$ be an another solution and put $w=v-H_{\alpha} \varphi$. Then $w$ is a solution of

$$
\begin{array}{ll}
(\alpha-A) w(x, \theta)=0, & (x, \theta) \in S^{0} \\
w(r \theta, \theta)=0, & (r \theta, \theta) \in \partial S,
\end{array}
$$

and so $w=G_{\alpha}^{\min } 0=0$, implying the uniqueness of the solution.

It follows from the definition that $H_{\alpha}$ is linear, non-negative, bounded and satisfies the property (iv). To prove (iii), we suppose that there exists the point $\left(x_{0}, \theta_{0}\right) \in S^{0}$ such that $H_{\alpha} \varphi\left(x_{0}, \theta_{0}\right)=\max _{(x, \theta) \in S} H_{\alpha} \varphi(x, \theta)>0 . H_{\alpha} \varphi$ satisfies

$$
A H_{\alpha} \varphi\left(x_{0}, \theta_{0}\right)=\alpha H_{\alpha} \varphi\left(x_{0}, \theta_{0}\right)>0 ;
$$

but the left hand side is non-positive, which is the contradiction. Hence $H_{\alpha} \varphi$ does not take a positive maximum in $S^{0}$. Moreover, $H_{\alpha} 1(x, \theta) \leq 1$, i.e., $\left\|H_{\alpha}\right\| \leq 1$. Thus the proof is completed.

Proposition 2. 1. Let $\left\{T_{t}, t \geq 0\right\}$ be the (strongly continuous and positive contraction) semigroup with the infinitesimal generator $A$. Then both of boundary points $(r \theta, \theta), \theta= \pm 1$, are regular.

Proof. Put

$$
G_{\alpha} f=\int_{0}^{\infty} e^{-\alpha t} T_{t} f d t \quad \text { for all } f \in C(S)
$$


Then $v=G_{\alpha} f-G_{\alpha}^{\min } f$ satisfies the following

$$
\begin{array}{ll}
(\alpha-A) v(x, \theta)=0, & (x, \theta) \in S^{0}, \\
v(r \theta, \theta)=G_{\alpha} f(r \theta, \theta), & (r \theta, \theta) \in \partial S .
\end{array}
$$

If $(r \theta, \theta)$ is an entrance or natural boundary, then $G_{\alpha} f(r \theta, \theta)$ must be zero by Theorem 2. 2. Hence

$$
f(r \theta, \theta)=\lim _{\alpha \rightarrow \infty} \alpha G_{\alpha} f(r \theta, \theta)=0,
$$

which contradicts the arbitariness of $f \in C(S)$.

If $(r \theta, \theta)$ is exit, then

$$
G_{\alpha} f=G_{\alpha}^{\min } f+H_{\alpha}\left[G_{\alpha} f\right]_{\partial S},
$$

where $\left[G_{\alpha} f\right]_{\partial S}$ is a restriction of $G_{\alpha} f$ to $\partial S$.

Hence, it follows from Theorem 2.1 and 2. 2 that

$$
\begin{aligned}
f(r \theta,-\theta) & =\lim _{\alpha \rightarrow \infty} \alpha G_{\alpha} f(r \theta,-\theta) \\
& =\lim _{\alpha \rightarrow \infty} \alpha G_{\alpha}^{\min } f(r \theta,-\theta)+\lim _{\alpha \rightarrow \infty} H_{\alpha}\left[\alpha G_{\alpha} f\right]_{\partial S}(r \theta,-\theta) \\
& =0 .
\end{aligned}
$$

This is also a contradiction. Thus $(r \theta, \theta)$ is regular, implying the proof.

We define the function $[u]_{\partial S}$ on $\partial S$ induced by the function $u$ on $S$ by

$$
[u]_{\partial S}(r \theta, \theta)=u(r \theta, \theta), \quad(r \theta, \theta) \in \partial S .
$$

Then we have

Proposition 2. 2. $C_{1(m)}(S)=\left\{v: v=G_{\alpha}^{\min } u+H_{\alpha} \varphi, u \in C(S), \varphi \in C(\partial S)\right\}$.

Proof. Let $u \in C_{1(m)}(S)$. Noting that $u-H_{\alpha}[u]_{\partial S}$ vanishes on $\partial S$ and $R\left(G_{\alpha}^{\min }\right)=\left\{u \in C_{1(m)}(S): u(r \theta, \theta)=0\right\}$, we can select the element $w$ of $C(S)$ such that $u-H_{\alpha}[u]_{\partial S}=G_{\alpha}^{\min } w$, i.e., $u \in\left\{v: v=G_{\alpha}^{\min } u+H_{\alpha} \varphi\right\}$. The converce is evidently true, since $G_{\alpha}^{\min } u$ and $H_{\alpha} \varphi$ belong to $C_{1(m)}(S)$. Thus we finish the proof.

Proposition 2. 3. Let $\zeta_{1}, \zeta_{-1} \in C(\partial S)$ be the functions defined as follows:

$$
\zeta_{1}(r \theta, \theta)= \begin{cases}1, & \text { if } \theta=1 \text { and }(r, 1) \text { is regular or exit, } \\ 0, & \text { if otherwise, }\end{cases}
$$




$$
\zeta_{-1}(r \theta, \theta)= \begin{cases}1, & \text { if } \theta=-1 \text { and }(-r,-1) \text { is regular or exit }, \\ 0, & \text { if otherwise. }\end{cases}
$$

Then it holds that

$$
H_{\alpha} \varphi(x, \theta)=\sum_{\vartheta= \pm 1} \varphi(r \vartheta, \vartheta) H_{\alpha} \zeta_{\vartheta}(x, \theta)
$$

Proof is evident on account of the uniqueness of the solution of the equation (2. 8).

§3. Semigroup on $\boldsymbol{C}(\boldsymbol{\partial S})$. In this section, we always assume that boundary points $(r \theta, \theta), \theta= \pm 1$, are regular, i.e. $|m(r \theta, \theta)|<\infty$. Let $A$ and $L$ be the operator defined by (1.1) and (1.2) in $\$ 1$. Then $L H_{\alpha} \varphi$ is welldefined for $\varphi \in C(\partial S)$ by noting that $H_{\alpha} \varphi \in C_{1(m)}(S)$. Thus we have

Lemma 3. 1. There exists a number $\lambda \geq 0$ such that

$$
\left(\lambda-L H_{\alpha}\right) \varphi(r \theta, \theta)=\psi(r \theta, \theta), \quad(r \theta, \theta) \in \partial S
$$

has a solution $\varphi \in C(\partial S)$ for each $\psi \in C(\partial S)$.

Proof. On account of Proposition 2. 3, the equation (3.1) can be changed to the form:

$$
\begin{aligned}
& \left\{\lambda H_{\alpha} \zeta_{1}(r \theta, \theta)-L H_{\alpha} \zeta_{1}(r \theta, \theta)\right\} \varphi(r, 1) \\
& +\left\{\lambda H_{\alpha} \zeta_{-1}(r \theta, \theta)-L H_{\alpha} \zeta_{-1}(r \theta, \theta)\right\} \varphi(-r,-1)=\psi(r \theta, \theta), \theta= \pm 1 .
\end{aligned}
$$

Hence, solving this system of linear equation, we can obtain the function $\varphi$. Thus the proof is finished.

Lemma 3. 2. If $\varphi \in C(\partial S)$ takes a positive maximum at $\left(r \theta_{0}, \theta_{0}\right) \in \partial S$, then

$$
L H_{\alpha} \varphi\left(r \theta_{0}, \theta_{0}\right) \leq 0 .
$$

Especially, $L H_{\alpha} 1(r \theta, \theta)<0$ for each $(r \theta, \theta) \in \partial S$.

Proof. Since $H_{\alpha} \varphi$ takes a positive maximum at $\left(r \theta_{0}, \theta_{0}\right)$ as a function on $S$ by Theorem 2. 2, we have

$$
\frac{\partial}{\partial n} H_{\alpha} \varphi\left(r \theta_{0}, \theta_{0}\right)=-\frac{d}{d m} H_{\alpha} \varphi\left(r \theta_{0}, \theta_{0}\right) \leq 0
$$

and

$$
\left[H_{\alpha} \varphi(y, \vartheta)-H_{\alpha} \varphi\left(r \theta_{0}, \theta_{0}\right)\right] \leq 0
$$


Therefore, noting that $H_{\alpha} \varphi=\varphi$ on $\partial S$ and $(\alpha-A) H_{\alpha} \varphi=0$, we have

$$
\begin{aligned}
L H_{\alpha} \varphi\left(r \theta_{0}, \theta_{0}\right)= & \Gamma\left(r \theta_{0}, \theta_{0}\right) \varphi\left(r \theta_{0}, \theta_{0}\right) \\
& +\delta\left(r \theta_{0}, \theta_{0}\right) \alpha H_{\alpha} \varphi\left(r \theta_{0}, \theta_{0}\right) \\
& +\mu\left(r \theta_{0}, \theta_{0}\right) \frac{\partial}{\partial n} H_{\alpha} \varphi\left(r \theta_{0}, \theta_{0}\right) \\
& +\int_{S}\left[H_{\alpha} \varphi(y, \vartheta)-H_{\alpha} \varphi\left(r \theta_{0}, \theta_{0}\right)\right] \nu_{\left(r \theta_{0}, \theta_{0}\right)}(d(y, \vartheta)) . \\
& \leq 0 .
\end{aligned}
$$

Since $H_{\alpha} 1$ is not a constant function and does not take a positive maximum in $S^{0}, \frac{\partial}{\partial n} H_{\alpha} 1(r \theta, \theta)<0$ and $\left[H_{\alpha} 1(y, \vartheta)-H_{\alpha} 1(r \theta, \theta)\right]<0$ for each $(y, \vartheta) \in S^{0}$. Hence, $L H_{\alpha} 1(r \theta, \theta)<0$. Thus the proof is completed.

Theorem 3. 1. For $\alpha>0, L H_{\alpha}$ is the infinitesimal generator of a semigroup on $C(\partial S)$.

We call the semigroup on $C(\partial S)$ with infinitesimal generator $L H_{\alpha}$ the semigroup on $C(\partial S)$ of order $\alpha$, and denote it by $\left\{S_{t}^{\alpha}, t \geq 0\right\}$. The Green operator of $\left\{S_{t}^{\alpha}, t \geq 0\right\}$ is denoted by

$$
K_{\lambda}^{\alpha} \varphi=\int_{0}^{\infty} e^{-\lambda t} S_{t}^{\alpha} \varphi d t, \lambda>0 .
$$

Proof. Noting that $L H_{\alpha}$ is closed because of the boundedness of $H_{\alpha}$ and the closedness of $\frac{\hat{o}}{\partial n} H_{\alpha}$, our result is directly obtained from [11, Theorem 1. 2] by virtue of Lemma 3.1-3. 2.

Corollary. For each $\psi \in C(\partial S)$,

$$
L H_{\alpha} \varphi=\varphi
$$

has a unique solution and hence $\left(L H_{\alpha}\right)^{-1}$ is well-defined on $C(\hat{\partial} S) .-\left(L H_{\alpha}\right)^{-1}$ is nonnegative and bounded.

We sometimes write $K_{0}^{\alpha}$ for $-\left(L H_{\alpha}\right)^{-1}$.

Proof. Since constant 1 belongs to $\mathfrak{D}\left(L H_{\alpha}\right)$ and $L H_{\star} 1(r \theta, \theta)<0$ at each $(r \theta, \theta) \in \partial S$ for $\alpha>0$ by Lemma 3.1, $L H_{\alpha}+k$ is the infinitesimal generator of a contraction semigroup on $C(\partial S)$ by [11, the corollary of Theorem 1. 1], where $-k=\max _{(r \theta, \theta) \in \partial S} L H_{\alpha} 1(r \theta, \theta)$. Thus, $-L H_{\alpha} \varphi=\left\{k-\left(L H_{\alpha}+k\right)\right\} \varphi=\psi$ has a unique solution $\varphi$ for each $\phi \in C(\partial S)$ by Hille-Yosida's theorem. - $\left(L H_{\alpha}\right)^{-1}$ is clearly non-negative and $\left\|-\left(L H_{\star}\right)^{-1}\right\| \leqq k^{-1}$, completing the proof. 
\$4. Construction of transport semigroup with regular boundary. In this section, we also assume that all of boundary points $(r \theta, \theta)$ $\langle\theta= \pm 1)$ are regular.

Let us denote by $\mathfrak{D}\left(A_{L-\lambda}\right)$ the subspace $\left\{u \in C_{1(m)}(S):(L-\lambda) u(r \theta, \theta)=0\right\}$ of $C(S)$. We consider the operators $A_{L-\lambda}$ and $G_{\alpha}^{\lambda}$ defined by

$$
\left.A_{L-\lambda}=A \mid \mathfrak{D}\left(A_{L-\lambda}\right) \quad \text { (a restriction of } A \text { to } \mathfrak{D}\left(A_{L-\lambda}\right)\right)
$$

and

$$
G_{\alpha}^{\lambda} u=G_{\alpha}^{\min } u+H_{\alpha} K_{\lambda}^{\alpha} L G_{\alpha}^{\min } u, u \in C(S) .
$$

Then we have the following:

Theorem 4. 1. Suppose that both of $(r \theta, \theta)(\theta= \pm 1)$ are regular, i.e., $|m(r \theta, \theta)|<\infty$. Then $A_{L-\lambda}$ is the infinitesimal generator of a semigroup on $C(S)$ for $\lambda \geq 0$. The Green operator is given by $G_{\alpha}^{\lambda}$.

We call this semigroup the transport semigroup with regular boundary.

To prove the theorem, we prepare a few lemmas.

LEMma 4.1. i) $G_{\alpha}^{\lambda} u$ is the unique solution of

$$
(\alpha-A) v=u
$$

contained in $\mathfrak{D}\left(A_{L-\lambda}\right)$.

ii) $G_{\alpha}^{\lambda}$ is non-nogative.

Proof. Since $G_{\alpha}^{\min }$ and $H_{\alpha}$ are the mappings from $C(S)$ to $C_{1(m)}(S)$ and

$$
\begin{aligned}
(\lambda-L) G_{\alpha}^{\lambda} u & =(\lambda-L) G_{\alpha}^{\min } u+(\lambda-L) H_{\alpha} K_{\lambda}^{\alpha} L G_{\alpha}^{\min } u \\
& =-L G_{\alpha}^{\min } u+\left(\lambda-L H_{\alpha}\right) K_{\lambda}^{\alpha} L G_{\alpha}^{\min } u \\
& =-L G_{\alpha}^{\min } u+L G_{\alpha}^{\min } u=0,
\end{aligned}
$$

therefore $G_{\alpha}^{\lambda}$ belongs to $\mathfrak{D}\left(A_{L-\lambda}\right)$. Thus $G_{\alpha}^{\lambda} u$ is the solution of $(\alpha-A) v=u$ in $\mathfrak{D}\left(A_{L-\lambda}\right)$, since $(\alpha-A) G_{\alpha}^{\lambda} u=(\alpha-A) G_{\alpha}^{\min } u+(\alpha-A) H_{\alpha} K_{\lambda}^{\alpha} L G_{\alpha}^{\min } u=u$.

Let $v$ be an another solution. Then $w=v-G_{\alpha}^{\lambda} u$ satisfies $(\alpha-A) w=0$. Hence $w=H_{\alpha}[w]_{\partial S}$. Further, $w$ satisfies $(\lambda-L) w(r \theta, \theta)=(\lambda-L) H_{\alpha}[w]_{\partial S}=0$. Thus it follows from Theorem 3.1 and its corollary that $w=0$, implying the uniqueness. 
$G_{\alpha}^{\lambda} \geq 0$ follows from evident inequalities $G_{\alpha}^{\min } \geq 0, H_{\alpha} \geq 0, K_{\lambda}^{\alpha} \geq 0$ and $L G_{\alpha}^{\min } \geq 0$. Thus we complete the proof.

LEMMA 4. 2.

$$
\left\|G_{\alpha}^{\lambda}\right\| \leq 1 / \alpha .
$$

Proof. We note that the following equality holds:

$$
\alpha G_{\alpha}^{\min 1}+H_{\alpha} 1=1+G_{\alpha}^{\min }(c-b),
$$

since the boundary values of the both sides coincide and we have the same value when we apply $\alpha-A$ on the both sides. Hence we have

$$
\begin{aligned}
& \left(\lambda-L H_{\alpha}\right) 1=\lambda-L H_{\alpha} 1 \\
& =\lambda+\alpha L G_{\alpha}^{\min } 1-L 1-L G_{\alpha}^{\min }(c-b) \\
& \geq \alpha \quad L \quad G_{\alpha}^{\min 1} \text {, }
\end{aligned}
$$

since $L 1+L G_{\alpha}^{\min }(c-b) \leq 0$ because of $b \geq c$. Then, applying $K_{\lambda}^{\alpha}$ on the both ends, we have

$$
1=K_{\lambda}^{\alpha}\left(\lambda-L H_{\alpha}\right) 1 \geq \alpha K_{\lambda}^{\alpha} L G_{\alpha}^{\min } 1 .
$$

This, combined with $\alpha G_{\alpha}^{\mathrm{m} ! \mathrm{n}} 1+H_{\alpha} 1 \leq 1$, imply

$$
\alpha G_{\alpha}^{\lambda} 1=\alpha G_{\alpha}^{\min 1}+\alpha H_{\alpha} K_{\lambda}^{\alpha} L \quad G_{\alpha}^{\min 1} \leq 1,
$$

completing the proof.

Lemma 4. 3. For any $\varepsilon>0$, there exists the function $w_{\theta}$ in $C_{1(m)}(S)$ such that:

i) $\quad\left\|w_{\theta}\right\|<\varepsilon$,

ii) $\quad A w_{\theta}(r \theta, \theta)=-1$,

iii) $\quad \frac{\partial}{\partial n} w_{\theta}(r \theta, \theta)=1$,

iv) $\quad \int_{S}\left[w_{\theta}(y, \vartheta)-w_{\theta}(r \theta, \theta)\right] \nu_{(r \theta, \theta)}(d(y, \vartheta))$

$$
\begin{aligned}
& =\int_{U_{(r \theta, \theta)}}\left[\zeta_{(r \theta, \theta)}(y, \vartheta)\right] \nu_{(r \theta, \theta)}(d(y, \vartheta))+0(1) \varepsilon, \text { if } \nu_{(r \theta, \theta)}(S)=\infty ; \\
& =0(1) \varepsilon, \text { if } \nu_{(r \theta, \theta)}(S)<\infty:
\end{aligned}
$$

where $\zeta_{(r \theta, \theta)}(y, \vartheta)$ and $U_{(r \theta, \theta)}$ are in (1. 3),

v) $\quad\left|A w_{\theta}(-r \theta,-\theta)\right|<\varepsilon$, 
vi)

$$
\frac{\partial}{\partial n} w(-r \theta,-\theta)=0
$$

vii)

$$
\left|\int_{S}\left[w_{\theta}(y, \vartheta)-w_{\theta}(-r \theta,-\theta)\right] \nu_{(-r \theta,-\theta)} d(y, \vartheta)\right|<\varepsilon .
$$

Proof. We assign $w_{\theta}$ by

$$
w_{\theta}(y, \vartheta)= \begin{cases}\int_{y}^{r \theta} \exp \left[-\int_{y}^{z} \frac{b(\xi, \theta)}{\theta a(\xi, \theta)} d \xi\right] \frac{f(z, \theta)}{\theta a(z, \theta)} d z, & \vartheta=\theta \\ 0 & \vartheta \neq \theta .\end{cases}
$$

We now choose the function $f$ in $C(S)$ which is 1 near $(r \theta, \theta)$ and 0 outside of the neighborhood of $(r \theta, \theta)$. Then $w_{\partial}$ is the function which is desired for us.

Lemma 4. 4. $\mathfrak{D}\left(A_{L-\lambda}\right)$ is dense in $C(S)$.

Proof. For $u \in C_{1(m)}(S)$, we set

$$
v=u+\alpha_{1} w_{1}+\alpha_{-1} w_{-1},
$$

where $w_{\theta}$ are in Lemma 4. 3. Then for any $\varepsilon>0$, we can select $\alpha_{1}, \alpha_{-1}$ such that $(\lambda-L) v(r \theta, \theta)=0$ and $\|u-v\|=0(1) \varepsilon$, completing the proof.

The proof of Theorem 4. 1 is the direct consequence of Hille-Yosida's Theorem by virtue of Lemma 4. 1, 4. 2 and 4. 3.

Combining the last theorem with Theorem 1.1 and Proposition 2.1, we have the following main theorem.

Theorem 4.2. Suppose that the operator $B$ on $C(S)$ with domain $\mathfrak{D}(B)$ is a restriction of $A$. Then $B$ is an infinitesimal generator of a (strongly continuous and positive contraction) semigroup on $C(S)$ if and only if

i) both of boundary points $(r \theta, \theta), \theta= \pm 1$, are regular,

ii) $\mathfrak{D}(B)=\mathfrak{D}\left(A_{L}\right)$.

§5. Transport semigroup with non-regular bounday. In this section, we show without precise argument that the transport semigroup with non-regular boundary can be constructed.

We introduce some subspaces and operators:

(5. 1) $\tilde{C}(S)=\{u \in C(S): \quad u(r \theta,-\theta)=0$, if $(r \theta, \theta)$ is an exit or natural 
boundary; $u(r \theta, \theta)=0$, if $(r \theta, \theta)$ is entrance or natural\},

(5. 2) $\tilde{C}(\partial S)=\{\varphi \in C(\partial S): \varphi(r \theta, \theta)=0$, if $(r \theta, \theta)$ is entrance or natural $\}$,

(5. 3) $\mathscr{D}\left(A_{L-\lambda}\right)=\left\{u \in C_{1(m)}(S): \cap \tilde{C}(S):(\lambda-L) u(r \theta, \theta)=0\right.$, if $(r \theta, \theta)$ is regular or exit\} $(\lambda \geq 0)$,

(5. 4) $\quad A_{L-\lambda}=A \mid \mathfrak{D}\left(A_{L-\lambda}\right) \quad(\lambda \geq 0)$,

(5. 5) $\quad H_{\alpha}(\alpha>0)$ : the mapping from $\tilde{C}(\partial S)$ to $\widetilde{C}(S)$ such that, for $\varphi \in \widetilde{C}(\partial S)$,

$$
\begin{array}{ll}
(\alpha-A) H_{\alpha} \varphi(x, \theta)=0, & (x, \theta) \in S^{0}, \\
H_{\alpha} \varphi(r \theta, \theta)=\varphi(r \theta, \theta), & (r \theta, \theta) \in \partial S,
\end{array}
$$

(5. 6) $\quad K_{\lambda}^{\alpha}(\lambda \geq 0, \alpha>0)$ : the mapping from $\widetilde{C}(\partial S)$ to $\widetilde{C}(\partial S)$ such that,

$$
\begin{aligned}
& \text { for } \varphi \in \widetilde{C}(\hat{o} S), \\
& \left(\lambda-L H_{\alpha}\right) K_{\lambda}^{\alpha} \varphi(r \theta, \theta)=\varphi(r \theta, \theta), \\
& \quad \text { if }(r \theta, \theta) \text { is regular or exit: } \\
& K_{\lambda}^{\alpha} \varphi(r \theta, \theta)=0, \\
& \quad \text { if otherwise, }
\end{aligned}
$$

(5. 7) $G_{\alpha}^{\lambda}(\lambda \geq 0, \alpha>0)$ : the mapping from $\tilde{C}(S)$ into itself defined by

$$
G_{\alpha}^{\lambda}=G_{\alpha}^{\min }+H_{\alpha} K_{\lambda}^{\alpha} L G_{\alpha}^{\min },
$$

Then we have the following:

Theorem 5. 1. For each $\lambda \geq 0, A_{L-\lambda}$ is the infinitesimal generator of a semigroup on $\tilde{C}(S)$. The Green operator is given by $G_{\alpha}^{\lambda}$.

We call this semigroup the transport semigroup with the regular, exit, entrance or natural boundary if $(r \theta, \theta)$ is regular, exit, entrance or natural, respectively.

Theorem 5.2. Suppose that the operator $B$ with domain $\mathbb{D}(B)$ on $\widetilde{C}(S)$ is a restriction of $A$.

In order that $B$ be the infinitesimal generator of a (strongly continuous and positive contraction) semigroup on $\tilde{C}(S)$ it is nessesary and sufficient that $\mathfrak{D}(B)=\mathfrak{D}\left(A_{L}\right)$.

\section{APPENDIX}

In this appendix, we consider the convergence of the transport process with regular boundaries to the Brownian motion with regular boundaries.* From now on, we always assume the following:

*) cf. [9] and [13]. 
( $\quad a(x, \theta) \equiv a \quad$ (positive constant)

$$
b(x, \theta) \equiv c(x, \theta) \equiv a^{2} .
$$

Then the operator $A$ becomes of the form:

$$
A u(x, \theta)=\theta a \frac{d}{d x} u(x, \theta)-a^{2} u(x, \theta)+a^{2} u(x,-\theta) .
$$

1. Concrete form and convergence of minimal Green operator $\boldsymbol{G}_{\alpha}^{\min }$ and hamonic operator $\boldsymbol{H}_{\alpha}$. On account of Theorem 3.1 and 3.2, $G_{\alpha}^{\min } f(f \in C(S))$ is the unique solution of

$$
\begin{gathered}
\alpha u(x, \theta)-\theta a \frac{d}{d x} u(x, \theta)+a^{2} u(x, \theta)-a^{2} u(x, \theta)=f(x, \theta),(x, \theta) \in S^{0}, \\
u(r \theta, \theta)=0, \quad(r \theta, \theta) \in \partial S,
\end{gathered}
$$

and $H_{\alpha} \varphi(\varphi \in C(\partial S))$ is the unique solution of

$$
\begin{gathered}
\alpha u(x, \theta)-\theta a \frac{d}{d x} u(x, \theta)+a^{2} u(x, \theta)-a^{2} u(x,-\theta)=0,(x, \theta) \in S^{0}, \\
u(r \theta, \theta)=\varphi(r \theta, \theta), \quad(r \theta, \theta) \in \partial S .
\end{gathered}
$$

By solving the equations (2) and (3), we have

Proposition 1. i) For $f \in C(S)$ and $\alpha>0$,

$$
\begin{aligned}
& G_{\alpha}^{\min } f(x, 1)= C e^{\beta x}+D e^{-\beta x} \\
&-\frac{e^{\beta x}}{2 \beta} \int_{-r}^{x}\left\{\left(1+\frac{\alpha}{a^{2}}+\frac{\beta}{a}\right) f(y, 1)+f(y,-1)\right\} e^{-\beta y} d y \\
&+\frac{e^{-\beta x}}{2 \beta} \int_{-r}^{x}\left\{f(y, 1)+\left(1+\frac{\alpha}{a^{2}}-\frac{\beta}{a}\right) f(y,-1)\right\} e^{\beta y} d y \\
& G_{\alpha}^{\min } f(x,-1)=C\left(1+\frac{\alpha}{a^{2}}-\frac{\beta}{a}\right) e^{\beta x}+D\left(1+\frac{\alpha}{a^{2}}+\frac{\beta}{a}\right) e^{-\beta x} \\
&-\left(1+\frac{\alpha}{a^{2}}-\frac{\beta}{2}\right) \frac{e^{\beta x}}{2} \int_{-r}^{x}\left\{\left(1+\frac{\alpha}{a^{2}}+\frac{\beta}{a}\right) f(y, 1)+f(y,-1)\right\} e^{-\beta y} d y \\
&+\left(1+\frac{\alpha}{a^{2}}+\frac{\beta}{a}\right) \frac{e^{-\beta x}}{2} \int_{-r}^{x}\left\{f(y, 1)+\left(1+\frac{\alpha}{a^{2}}-\frac{\beta}{a}\right) f(y,-1)\right\} e^{\beta y} d y,
\end{aligned}
$$

where $\beta=\sqrt{2 \alpha+\frac{\alpha^{2}}{a^{2}}}>0, C$ and $D$ are determined by 


$$
\begin{aligned}
& e^{\beta r} C+e^{-\beta r} D \\
= & \frac{e^{\beta r}}{2} \int_{-r}^{r}\left\{\left(1+\frac{\alpha}{a^{2}}+\frac{\beta}{a}\right) f(y, 1)+f(y,-1)\right\} e^{-\beta y} d y \\
- & \frac{e^{-\beta r}}{2} \int_{-r}^{r}\left\{f(y, 1)+\left(1+\frac{\alpha}{a^{2}}-\frac{\beta}{a}\right) f(y,-1)\right\} e^{\beta y} d y, \\
& \left(1+\frac{\alpha}{a^{2}}-\frac{\beta}{a}\right) e^{-\beta r} C+\left(1+\frac{\alpha}{a^{2}}+\frac{\beta}{a}\right) e^{\beta r} D=0 .
\end{aligned}
$$

ii) For $\varphi \in C(\partial S)$ and $\alpha>0$,

$$
\begin{aligned}
& H_{\alpha} \varphi(x, 1)=E e^{\beta x}+F e^{-\beta x} \\
& H_{\alpha} \varphi(x,-1)=E\left(1+\frac{\alpha}{a^{2}}-\frac{\beta}{a}\right) e^{\beta x}+F\left(1+\frac{\alpha}{a^{2}}+\frac{\beta}{a}\right) e^{-\beta x},
\end{aligned}
$$

where $\beta=\sqrt{2 \alpha+\frac{\alpha^{2}}{a^{2}}}>0, E$ and $F$ are determined by

$$
\begin{aligned}
& e^{\beta r} E+e^{-\beta r} F=\varphi(r, 1) \\
& \left(1+\frac{\alpha}{a^{2}}-\frac{\beta}{a}\right) e^{-\beta r} E+\left(1+\frac{\alpha}{a^{2}}+\frac{\beta}{a}\right) e^{\beta r} F=\varphi(-r,-1)
\end{aligned}
$$

Now let $\frac{1}{2} \Delta$ be the Laplacian operator on $I=[-r, r]$, i.e.,

$$
\frac{1}{2} \Delta u(x)=\frac{1}{2} \frac{d^{2}}{d x^{2}} u(x)
$$

As is well-known, the minimal Green operator $G_{\alpha}^{\Delta \min }$ and the harmonic operator $H_{\alpha}^{\Delta}$, corresponding to $\frac{1}{2} \Delta$, is given as follows:

$$
\begin{aligned}
G_{\alpha}^{\Delta, \min } f(x) & =C^{\Delta} e^{\sqrt{2 \alpha} x}+D^{\Delta} e^{-\sqrt{2 \alpha} x} \\
& -\frac{e^{\sqrt{2 \alpha} x}}{\sqrt{2 \alpha}} \int_{-r}^{x} f(y) e^{-\sqrt{2 \alpha} y} d y+\frac{e^{-\sqrt{2 \alpha} x}}{\sqrt{2 \alpha}} \int_{-r}^{x} f(y) e^{\sqrt{2 \alpha} y} d y, \\
x \in & (-r, r), \quad f \in C(I),
\end{aligned}
$$

where $C^{\boldsymbol{\Delta}}$ and $D^{\boldsymbol{\Delta}}$ are determined by

$$
\begin{aligned}
& e^{\sqrt{2 \alpha} r} C^{\Delta}+e^{-\sqrt{2 \alpha} r} D^{\Delta} \\
= & \frac{e^{\sqrt{2 \alpha} r}}{\sqrt{2 \alpha}} \int_{-r}^{r} f(y) e^{-\sqrt{2 \alpha} y} d y-\frac{e^{-\sqrt{2 \alpha} r}}{\sqrt{2 \alpha}} \int_{-r}^{r} f(y) e^{\sqrt{2 \alpha} y} d y,
\end{aligned}
$$




$$
e^{-\sqrt{2 \alpha}} r C^{\Delta}+e^{\sqrt{2 \alpha} r} D^{\Delta}=0
$$

$$
H_{\alpha} \varphi(x)=E^{\Delta} e^{\sqrt{2 \alpha} x}+F^{\Delta} e^{-\sqrt{2 \alpha} x},
$$

where $E^{\Delta}$ and $F^{\Delta}$ are determined by

$$
\begin{aligned}
& e^{\sqrt{2 \alpha} r} E^{\Delta}+e^{-\sqrt{2 \alpha} r} F^{\Delta}=\varphi(r), \\
& e^{-\sqrt{2 \alpha} r} E^{\Delta}+e^{\sqrt{2 \alpha} r} F^{\Delta}=\varphi(-r), \\
& \varphi \in C(\partial I), \quad \partial I=\{r\} \cup\{-r\} .
\end{aligned}
$$

We now define the mapping from $C(I)(C(\partial I))$ to $C(S)(C(\partial S))$ as the following:

$$
\begin{aligned}
P: f(x) & \rightarrow(P f)(x, \theta)=f(x) \\
\varphi(r \theta) \rightarrow(P \varphi)(r \theta, \theta) & =\varphi(r \theta) .
\end{aligned}
$$

Then we have

Proposition 2. i) For $f \in C(I)$, it holds that, as $a \rightarrow \infty *$,

$$
G_{\alpha}^{\min } P f \rightarrow P G_{\alpha}^{\Delta, \min f}
$$

2)

$$
\frac{d}{d x} G_{\alpha}^{\min } P f \rightarrow \frac{d}{d x} P G_{\alpha}^{4, \min f}
$$

3) moreover, if $f \in C_{1}(I)^{* *)}$,

$$
\frac{d^{2}}{d x^{2}} G_{\alpha}^{\min } P f \rightarrow \frac{d^{2}}{d x^{2}} P G_{\alpha}^{\Delta, \min } f .
$$

ii) For $\varphi \in C(\partial I)$, it holds that, as $a \rightarrow \infty$,

$$
H_{\alpha} P \varphi \rightarrow P H_{\alpha}^{\Delta} \varphi
$$

2)

$$
\frac{d}{d x} H_{\alpha} P \varphi \rightarrow \frac{d}{d x} P H_{\alpha}^{\Delta} \varphi
$$

3)

$$
\frac{d^{2}}{d x^{2}} H_{\alpha} P \varphi \rightarrow \frac{d^{2}}{d x^{2}} P H_{\alpha}^{\Delta} \varphi
$$

§2. Convergence of $\boldsymbol{K}_{\lambda}^{\alpha}$. Let $L^{\Delta}$ be the operator on $\partial I$ as follows:

$$
L^{\Delta} u(r \theta)=0
$$

*) $a$ is the coefficient in the definition of $A$.

**) $C_{1}(I)$ is the subspace of $C(I)$ of continuously differenciable functions. 


$$
\begin{aligned}
L^{\Delta} u(r \theta)= & \gamma(r \theta) u(r \theta)+\delta(r \theta) \frac{1}{2} \Delta u(r \theta)+\mu(r \theta) \frac{\partial}{\partial n} u(r \theta) \\
& +\int_{I}\left[(u(y)-u(r \theta)] \nu_{r_{\theta}}(d y),\right.
\end{aligned}
$$

where $\gamma, \delta,-\mu$ are non-positive and $\nu$ is a $\sigma$-finite measure on $I$ satisfying:

$$
\begin{aligned}
& \nu_{r_{\theta}}\{(r \theta)\}=0, \\
& \int_{I}(1 \wedge(r+y) \wedge(r-y))_{\nu_{\theta}}(d y)<\infty, \\
& \nu_{r_{\theta}}(I)=\infty, \text { if } \gamma=\delta=\mu=0 .
\end{aligned}
$$

Then there exists the Green operator $K_{\lambda}^{\Delta_{\alpha}}(\lambda \geq 0)$ corresponding to $L^{\Delta} H_{\alpha}^{\Delta}$ (see. §3).

Let $L$ be the boundary condition defined in $\$ 1$. We assume that the operators $L$ and $L^{\Delta}$ satisfy the followings:

$$
\begin{aligned}
& r(r \theta)=\gamma(r \theta, \theta), \\
& \delta(r \theta)=\delta(r \theta, \theta), \\
& \mu(r \theta)=\mu(r \theta, \theta), \\
& \nu_{r_{\theta}}(d y)=\nu_{(r \theta, \theta)}(d(y, \theta)), \\
& \nu_{(r \theta, \theta)}([-r, r] \times\{-\theta\})=0 .
\end{aligned}
$$

Then we have from Proposition 2

Lemma 1. $L H_{\alpha} P \varphi \rightarrow P L^{\Delta} H_{\alpha}^{\Delta} \varphi$ as $a \rightarrow \infty$ for $\varphi \in C(\partial I)$.

Thus, by virtue of Trotter's theorem [12, Theorem 5. 2], it follows

LeMma 2. For $\varphi \in C(\partial I)$ and $\lambda \geq 0$,

$$
K_{\lambda}^{\alpha} P \varphi \rightarrow P K_{\lambda}^{\Delta, \alpha} \varphi \quad \text { as } \quad a \rightarrow \infty .
$$

\$3. Convergence of transport process. Suppose that $C_{2}(I)$ is the subspace $C(I)$ of each of which is twice continuously differentiable: for $\lambda \geq 0$, let $D\left(\Delta_{L} \Delta_{-\lambda}\right)$ be the subspace $\left\{u \in C_{2}(I):\left(L^{\Delta}-\lambda\right) u(r \theta)=0, \theta= \pm 1\right\}$ and $\frac{1}{2} \Delta_{L} \Delta_{-\lambda}$ be a restriction of the Laplacian operator $\frac{1}{2} \Delta$ to $D\left(\Delta_{L} \Delta_{-\lambda}\right)$. By the same argument in $\$ 4$, we have

PROPOSITION 3. $\frac{1}{2} \Delta_{L_{-\lambda}}$ is the infinitesimal generator of a semigroup on 
$C(I)$. The Green operator is given by

$$
G_{\alpha}^{\lambda}=G_{\alpha}^{\Delta, \min }+H_{\alpha}^{\Delta} K_{\lambda}^{\Delta \alpha} L^{\Delta} G_{\alpha}^{\Delta, \min } .
$$

Combining this with Proposition 2 and Lemma 2, we have

Lemma 3. For $u \in C(I), \alpha>0$ and $\lambda \geq 0$,

$$
G_{\alpha}^{\lambda} P u \rightarrow P G_{\alpha}^{\Delta, \lambda} u \quad \text { as } a \rightarrow \infty .
$$

Thus we obtain the following main result.

Theorem. Suppose that both conditions $(\mathscr{A})$ and $(\mathscr{L})$ are satisfied. Then the transport $\left\{T_{t}, t \geq 0\right\}$ semigroup with regular boundary condition $(L)$ converges, in Trotter's sence, to the semigroup $\left\{T_{t}^{\Delta}, t \geq 0\right\}$ of the Brownian motion with regular boundary condition $\left(L^{\Delta}\right)$ when $a$ in condition ( $\mathscr{A}$ ) grows up indefinitely, i.e.,

$$
T_{t} P f \rightarrow P T_{t}^{\Delta} f \quad \text { as } a \rightarrow \infty \text { for } f \in C(I) .
$$

\section{REFERENCES}

[1] P. Courrege, Problemes aux limites elliptiques et principe du maximum, Sém. Bourbaki, $1965 / 66, n^{0} 302$.

[2] E.B. Dynkin, Markov processes, Springer (1964).

[ 3 ] W. Feller, The parabolic differential equations and the associated semigroup of transformations, Ann. Math. 55 (1952), 468-519.

[4] — Diffusion processes in one dimension, Trans. Amer. Math. Soc., 77 (1954), $1-31$.

[ 5 ] — , On second order differential operators, Ann. Math., 61 (1955), 90-105.

[6] - Generalized second order differential operators and their lateral conditions. Illinois J. Math., 1 (1957), 459-504.

[7] M. Fukushima, K. Ito and S. Watanabe, Diffusion processes, Seminar on Prob., 3 (1960) (Japanese).

[ 8 ] N. Ikeda, On the construction of two dimensional diffusion processes satisfying Wentzell's boundary conditions and its application to boundary value problems, Mem. Coll. Sci. Univ. Kyoto, Ser. A, 33 (1961), 367-427.

[9] N. Ikeda and H. Nomoto, Transport branching processes, Seminar on Prob., 25 (1966), 63-104 (Japanese).

[10] K. Ito and H.P. McKean, Jr, Diffusion Processes and their sample paths, Springer (1965).

[11] K. Sato and T. Ueno, Multi-dimensional diffusion and the Markov process on the boundary, J. Math. Kyoto Univ., 4 (1965), 529-605.

[12] H:F. Trotter, Approximation of semigroups on oprators, Pacific Jour. Math., 8 (1958), 887-919.

[13] To. Watanabe, Approximation of uniform transport process on a finite interval to Brownian motion, Nagoya Math. J., 32 (1968), 297-314.

[14] A.D. Wentzell, On lateral conditions for multi-dimensional diffusion processes, Teor. Veroyat. Primen., 4 (1959), 172-185 (Russian). 
[15] - General boundary problems connected with diffusion processes, Uspehi. Mat. Nauk, 15 (1960), 202-204 (Russian).

[16] - Semigroups of operaters corresponding to a generalized differential operator of second order, DAN SSSR, 111, 2 (1956), 269-272 (Russian).

Department of Applied Physics, Nagoya University 